\title{
Phosphorylated S6 as an immunohistochemical biomarker of vulvar intraepithelial neoplasia
}

\author{
Alvaro P Pinto ${ }^{1}$, Martin Degen ${ }^{2}$, Patricia Barron ${ }^{2}$, Christopher P Crum ${ }^{1}$ and \\ James G Rheinwald ${ }^{2}$ \\ ${ }^{1}$ Division of Women's and Perinatal Pathology, Department of Pathology, Brigham and Women's Hospital, \\ Boston, MA, USA and ${ }^{2}$ Department of Dermatology, Brigham and Women's Hospital, Harvard Skin Disease \\ Research Center, Boston, MA, USA
}

\begin{abstract}
As life expectancy lengthens, cases of non-viral-associated vulvar squamous cell carcinoma and its precursor lesion, so-called differentiated vulvar intraepithelial neoplasia (VIN), continue to increase in frequency. Differentiated VIN often is difficult to recognize and failure to detect it before invasion results in morbidity and mortality. Thus, identification of a reliable biomarker for this type of lesion would be of great clinical benefit. Our recent studies have identified activation (ser235/236 phosphorylation) of ribosomal protein S6 (p-S6) in basal epithelial cells as an event that precedes and accompanies laminin $\gamma^{2}$ overexpression in most preinvasive oral dysplasias. To test this as a potential biomarker of vulvar dysplasia, we immunostained seven differentiated VINs and nine papillomavirusrelated 'classic' VINs, most of which were associated with carcinoma, for p-S6. All carcinomas, all differentiated VINs, and most classic VINs contained regions of p-S6 staining in the basal layer, whereas basal and parabasal cells of normal vulvar epithelium and hyperplastic and inflamed lesions lacking cellular atypia were p-S6 negative. Laminin $\gamma^{2}$ was expressed in a subset of VINs, always occurring within basal p-S6 positive regions, as we had found previously for oral dysplasias. Lichen sclerosus is considered a potential precursor of vulvar carcinoma. Two lichen sclerosus lesions of patients with a concurrent carcinoma and one of six lichen sclerosus lesions without atypia or known concurrent carcinoma were basal p-S6 positive. In summary, there is a distinct difference in p-S6 basal cell layer staining between benign and neoplastic vulvar squamous epithelium, with consistent staining of differentiated VIN and of some lichen sclerosus lesions. These results support further studies to assess the potential of p-S6 as a biomarker to identify vulvar lesions at risk of progressing to invasive cancer.

Modern Pathology (2013) 26, 1498-1507; doi:10.1038/modpathol.2013.85; published online 14 June 2013
\end{abstract}

Keywords: laminin; lichen sclerosus; S6; squamous cell carcinoma; VIN

Vulvar squamous cell carcinoma accounts for $5 \%$ of female reproductive and genital tract cancers in the United States, with 4500 women initially diagnosed and 1000 dying of this cancer in $2012 .^{1}$ There are two widely recognized oncogenic pathways to vulvar carcinoma. ${ }^{2}$ The first, which occurs in $40 \%$ of cases, is initiated by infection by the high-risk human papillomavirus HPV16 and leads to the development of the so-called warty and basaloid types of vulvar carcinoma. ${ }^{3,4}$ HPV16-initiated carcinogenesis involves genomic integration of the viral oncogenes encoding E6 and E7, which inactivate and degrade

Correspondence: Dr JG Rheinwald, PhD, Department of Dermatology, Brigham and Women's Hospital, Harvard Skin Disease Research Center, 77 Avenue Louis Pasteur, HIM 664, Boston, MA 02115, USA.

E-mail: jimrheinwald@gmail.com

Received 28 November 2012; revised 13 April 2013; accepted 18 April 2013; published online 14 June 2013 the tumor suppressor genes $\mathrm{pRb}$ and p53, respectively. As a consequence, the cell cycle is deregulated, p16 ${ }^{\mathrm{INK} 4 \mathrm{~A}}$ expression is induced but has no growth inhibitory effect on the pRb-inactive cells, and hyperplasia and dysplasia ensue. ${ }^{5}$ HPV16associated carcinomas are preceded by dysplastic lesions termed 'classic' vulvar intraepithelial neoplasia (VIN). Classic VINs tend to be multifocal, to arise in premenopausal women, and, with acquisition of further genetic changes, may progress to invasive carcinoma. ${ }^{6}$

The second pathway leading to vulvar carcinoma results from genetic and epigenetic changes in the absence of papillomavirus infection. Genetic alterations in the precursor lesions, so-called 'differentiated' VIN, include allelic imbalances, microsatellite instability, and p53 mutations. ${ }^{7-9}$ Differentiated VINs tend to be unifocal, to affect older women, and to precede the majority $(60 \%)$ of vulvar carcinomas. ${ }^{3,10}$ Histopathologic changes in 
differentiated VINs can be very subtle and there are no reliable biomarkers to identify them with certainty, making their detection and diagnosis challenging. Vulvar carcinoma may also arise within nondysplastic or minimally dysplastic lichen sclerosus lesions, so may go unheralded until invasive carcinoma has developed. ${ }^{11-14}$

The incidence of VIN has increased $>4$-fold since the early 1970s. ${ }^{15}$ Whereas classic VIN and its associated carcinoma are expected to decrease substantially in the future as the result of vaccination against high-risk papillomaviruses, ${ }^{16}$ the incidence of differentiated VIN and its associated carcinoma is rising, likely owing to the increasing lifespan of our population. Immunohistochemical analysis for abnormal patterns of $\mathrm{p} 16^{\mathrm{INK} 4 \mathrm{~A}}$, p53, and MIB1/Ki-67 expression, separately or as a panel, can distinguish classic from differentiated VIN but these markers often fail to identify the latter. ${ }^{17-20}$ We therefore sought to find a more reliable biomarker for differentiated VIN.

Abnormal, overexpression of the $\gamma^{2}$ subunit of the stratified epithelial basement membrane protein Laminin-332 was identified long ago as an immunohistochemically detectable characteristic of many types of invasive carcinoma. ${ }^{21-23}$ More recently it was found that laminin $\gamma^{2}$ overexpression begins at the preinvasive dysplastic stage of neoplastic progression in the oral epithelium and interfollicular epidermis. ${ }^{24,25}$ Squamous cell carcinomas of the interfollicular epidermis, oral cavity, oropharynx, and lung very frequently have gene mutations and amplifications that hyperactivate their EGFR/ERK signaling pathway. $^{26-28}$ Our recent study identified EGFR/ERK signal pathway hyperactivation as necessary and sufficient to drive laminin $\gamma^{2}$ overexpression in cultured human premalignant epithelial and squamous cell carcinoma cell lines and also found that the HPV16 E6 oncoprotein also induces laminin $\gamma^{2}$ overexpression. ${ }^{29}$ Laminin $\gamma^{2}$ overexpression proved to require the ERK-activated RSK kinase and to correlate with increased phosphorylation of the translation factor eIF4B at S442 and of ribosomal protein S6 at S235/236. ${ }^{29}$ Increased S6(S235/236) phosphorylation (p-S6) proved to be detectable immunohistochemically in the basal cell layer of oral dysplasias and squamous cell carcinomas, preceding and accompanying laminin $\gamma^{2}$ overexpression. ${ }^{29}$ We therefore sought in the present study to determine whether p-S6 in the basal cell layer is a typical feature of VIN and other potentially premalignant vulvar lesions.

\section{Materials and methods}

\section{Cases Examined}

Sixteen cases of vulvar dysplasia were selected from the files of the Brigham and Women's Hospital Department of Pathology. The formalin-fixed and paraffin-embedded selected specimens included seven differentiated VINs, six of which were associated with invasive carcinoma, and nine classic VINs, six of which were associated with invasive carcinoma. VINs were classified as classic or differentiated independently by two of the authors who are gynecologic pathology specialists (A.P.P. and C.P.C.), using conventional histopathological criteria ${ }^{30}$ and their classifications concurred in all cases. For comparison, 13 vulvar lesions judged by morphologic criteria not to be dysplastic, 12 of which were not associated with VIN or carcinoma at the time of biopsy, were selected from Pathology Department files. These 13 lesions were evaluated and classified independently by one of us (C.P.C.) and by Brigham and Women's Hospital staff pathologists who examined the specimens to write the original clinical pathology reports. Their diagnoses concurred in 12 of the cases, with the exception being a lichen sclerosus lesion that was scored as non-dysplastic by C.P.C. and mildly dysplastic in the original pathology report.

\section{Antibodies}

Antibodies used for immunostaining were: p-S6 (S235/236) (rabbit monoclonal antibody clone 91B2; Cell Signaling Technology, Danvers, MA, USA) at 1:100 dilution and laminin $\gamma^{2}$ (mouse monoclonal antibody clone D4B5; Millipore, Billerica, MA, USA) at 1:100 dilution.

\section{Immunohistochemical Staining}

Four-micrometer sections were stained with hematoxylin and eosin (H\&E) and immunostained for p-S6 and laminin $\gamma^{2}$. In order to improve retention of antigens for immunostaining, ${ }^{24}$ sections were not 'baked' at $50{ }^{\circ} \mathrm{C}$ after cutting as is the customary practice in pathology labs. Instead, they were warmed at $37^{\circ} \mathrm{C}$ for $30 \mathrm{~min}$ before deparaffinizing in xylene and rehydrating through 100, 95, and $70 \%$ ethanol and PBS. Antigen retrieval was carried out in $0.1 \mathrm{mM}$ citrate buffer ( $\mathrm{pH}$ 6.0) by autoclaving for $15 \mathrm{~min}$ at $120^{\circ} \mathrm{C}$ under pressure of 23 p.s.i. Endogenous peroxidase activity was blocked by incubation in $0.3 \%$ hydrogen peroxide in methanol for $10 \mathrm{~min}$. After a $20 \mathrm{~min}$ blocking step in $10 \%$ horse serum (Invitrogen, Carlsbad, CA, USA), sections were incubated with primary antibodies either for $1 \mathrm{~h}$ at room temperature or overnight at $4{ }^{\circ} \mathrm{C}$ (these methods proved to yield the same immunostaining patterns), followed by incubation with biotinylated secondary antibody (1:100) for $1 \mathrm{~h}$ and avidin/biotin/peroxidase complex (ABC; Vectastatin Elite ABC kit, Vector Laboratories, Burlingame, CA, USA) for $45 \mathrm{~min}$, with three PBS rinses between each step. Bound antibody was visualized with 3,3'-diaminobenzidine as a chromogen (EnVisionSystem, DAKO, Glostruo, Denmark). 
Some of the specimens were counterstained with Harris hematoxylin (Fisher Scientific, Kalamazoo, MI, USA). Slides were coverslip-mounted with Permount (Fisher Scientific) and examined with a NIKON Eclipse TE2000-S microscope (Nikon, Melville, NY, USA) fitted with an Insight QE SPOT camera (SPOT Imaging Solutions, Sterling Heights, MI, USA). Images were captured using SPOT software.

Some of the sections analyzed also contained regions of histologically normal epithelium, which served as a negative control to assess background staining. Immunostaining results were evaluated and photographed by two of the authors (A.P.P. and J.G.R.). P-S6 is present in the upper layers and absent in the basal and parabasal layers of normal oral epithelium, but frequently is present in cells of the basal layer in oral dysplasias and squamous cell carcinomas. ${ }^{29}$ Laminin $\gamma^{2}$ is undetectable in normal basal cells but frequently is present in the cytoplasm of basal cells in oral and epidermal dysplasias. ${ }^{24,29}$ In the present study, p-S6 and laminin $\gamma^{2}$ were scored as positive when present in any basal cells in the section, regardless of the number or proportion of positive cells.

\section{Results}

\section{P-S6 Immunostaining in Normal Vulvar Epithelium, Vulvar Carcinoma, and Classic and Differentiated VIN}

Our recent identification of p-S6(S235/236) as a readout for EGFR/ERK signal pathway hyperactivation in neoplastic oral epithelium and the presence of p-S6positive basal cells in oral dysplastic lesions and squamous cell carcinomas ${ }^{29}$ provided the rationale to examine VINs and vulvar carcinomas for this biomarker. We immunostained seven differentiated VINs, six of which were associated with carcinoma, and nine classic VINs, six of which were associated with carcinoma, for p-S6. Some of the specimens also contained histologically normal epithelium. As we
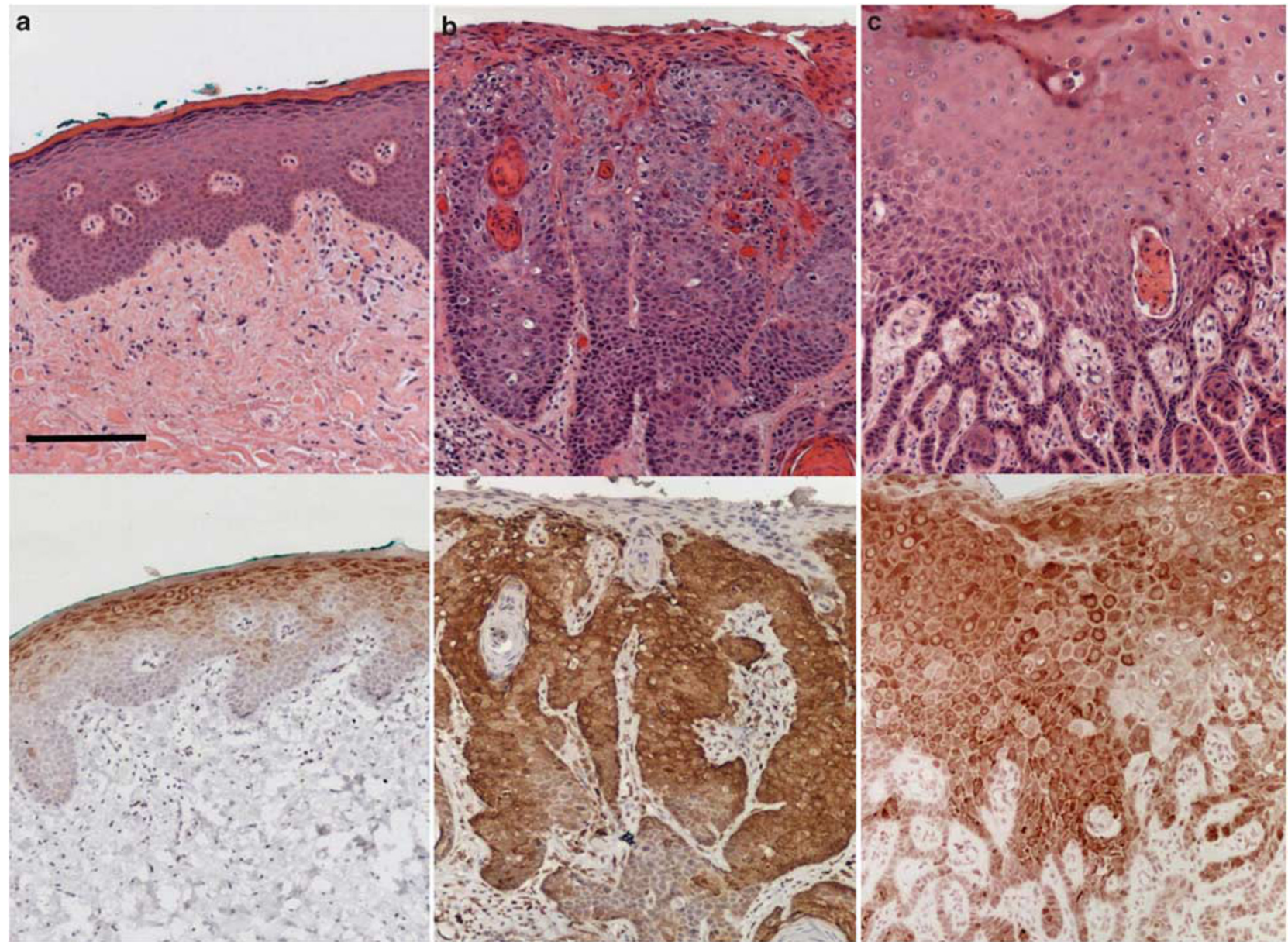

Figure 1 P-S6 immunostaining patterns in normal vulvar epithelium and carcinoma. H\&E staining (top) and p-S6(S235/236) immunostaining (bottom) of (a) normal vulvar epithelium from case 10, (b) a carcinoma that was associated with a classic VIN, case 14, (c) a carcinoma that was associated with a differentiated VIN, case 1. Bar in a is $200 \mu \mathrm{m}$. Note that p-S6 positive cells are confined to terminally differentiated suprabasal layers in the normal epithelium shown in a, whereas basal and parabasal cells are p-S6 positive in many areas of the carcinomas shown in $\mathbf{b}$ and $\mathbf{c}$. 
found previously for normal oral epithelium, the basal cells and first several suprabasal cell layers of normal vulvar epithelium were p-S6 negative, with more superficial terminally differentiated cells variably positive (Figure 1a). In contrast, p-S6 positive basal cells were frequent in all 12 carcinomas present in our specimen set (Figures $1 \mathrm{~b}$ and $\mathrm{c}$ ).

As summarized in Table 1, p-S6 was detectable in basal cells of seven of the nine classic VINs (Figures $2 \mathrm{a}-\mathrm{C})$ and in all seven differentiated VINs examined (Figures 2d-f). This result was consistent with our recent analysis of oral dysplasias. ${ }^{29} \mathrm{P}-\mathrm{S} 6$ positive regions of VINs typically showed full-thickness cytoplasmic staining (Figure 2). Regions identified as dysplastic (VIN) by conventional H\&E histopathologic morphology in each specimen were analyzed for the percentage of the dysplastic region that contained p-S6 positive basal cells. This ranged from 15 to $65 \%$ for differentiated VINs and from 0 to $60 \%$ for classic VINs (Table 1 ).

\section{P-S6 in Nondysplastic Vulvar Epithelial Lesions}

It is widely believed that vulvar carcinoma arises with increased probability from lichen sclerosus lesions, which may not display apparent dysplasia or atypia. ${ }^{11-14}$ In our survey of the 16 VINs described above, we found p-S6-positive basal cells in a region of lichen sclerosus without morphologic atypia adjacent to a differentiated VIN and invasive carcinoma in case 5 (data not shown). This observation prompted us to examine an additional case of lichen sclerosus associated with carcinoma plus a set of 12 vulvar biopsies that had been classified histopathologically as nondysplastic and were not associated with apparent VIN or carcinoma at the time of biopsy. These included six lichen sclerosus lesions and six hyperplastic and/or inflamed lesions (Table 2).

As we had found for wounds and ulcers in nondysplastic oral epithelium, ${ }^{29}$ basal cells at the edge of a wound in histologically normal vulvar epithelium were p-S6 positive (Figure 3a. (In this connection, it is important to note that in our analysis of p-S6 staining of VINs above we did not score as positive any regions that were within 20 cells of the edge of a wound or ulcer). Ulcerated regions of two inflamed, non-dysplastic lesions also contained basal p-S6 positive cells (Table 2, Figure $3 \mathrm{~b}$ ). The other hyperplastic and inflamed lesions were basal p-S6 negative (Table 2, Figure 3c).

The lichen sclerosus lesion of a patient that had carcinoma at the time of biopsy had an extensive region of basal p-S6 positive cells (Figure 3d), as did one of the six non-carcinoma-associated lichen sclerosus lesions (Figure 3e). The other lichen sclerosus lesions examined were basal p-S6 negative (Figure 3f). Importantly, regions of lichen sclerosus lesions in which the epithelium had separated from the underlying connective tissue, as commonly seen
Table 1 Summary of immunostaining results for VIN lesions

\begin{tabular}{|c|c|c|c|c|c|}
\hline Case \# & $\begin{array}{l}\text { Patient } \\
\text { age } \\
\text { (years) }\end{array}$ & $\begin{array}{l}\text { Histopathologic } \\
\text { classification }\end{array}$ & $\begin{array}{l}\text { VIN length in } \\
\text { mm, }(\% \text { with } \\
\text { basal p-S6) }\end{array}$ & $L a m \gamma^{2}$ & $\begin{array}{l}\text { Tumor in } \\
\text { specimen }\end{array}$ \\
\hline 1 & 81 & dVIN & $20(65)$ & $\mathrm{P}$ & SCC 2 \\
\hline 2 & 82 & dVIN & $4.2(60)$ & ND & SCC 2 \\
\hline 3 & 55 & dVIN & $10(60)$ & $\mathrm{N}$ & SCC \\
\hline 4 & 69 & dVIN & $35(25)$ & $\mathrm{P}$ & SCC 1 \\
\hline 5 & ND & $\begin{array}{l}\text { dVIN with lichen } \\
\text { sclerosus }\end{array}$ & $7.5(65)$ & $\mathrm{N}$ & SCC \\
\hline 6 & ND & dVIN & $18(15)$ & $\mathrm{N}$ & No tumor \\
\hline 7 & 65 & dVIN & $40(50)$ & $\mathrm{P}$ & SCC \\
\hline 8 & 65 & cVIN3 & $10(60)$ & $\mathrm{P}$ & No tumor \\
\hline 9 & 52 & cVIN3 & $28(35)$ & $\mathrm{P}$ & SCC \\
\hline 10 & 56 & cVIN3 & $18(5)$ & $\mathrm{N}$ & SCC \\
\hline 11 & 93 & cVIN3 & $30(60)$ & $\mathrm{N}$ & SCC sup. \\
\hline 12 & ND & cVIN3 & $19(0)$ & $\mathrm{N}$ & No tumor \\
\hline 13 & ND & cVIN3 & $30(60)$ & ND & SCC \\
\hline 14 & ND & cVIN2/3 & $27(0)$ & ND & SCC \\
\hline 15 & ND & cVIN2 & $10(5)$ & ND & No tumor \\
\hline 16 & ND & cVIN3 & $8(12)$ & $\mathrm{N}$ & SCC sup. \\
\hline
\end{tabular}

Abbreviations: cVIN, classic VIN; dVIN, differentiated VIN; N, no laminin $\gamma^{2}$ positive cells; ND, not determined; P, some laminin $\gamma^{2}$ positive cells; SCC, squamous cell carcinoma; sup, superficially invasive.

Sections were examined microscopically to determine the total 'length' of dysplastic areas and the percent of the dysplastic regions that were basal layer p-S6 positive. Classic VINs and carcinomas were classified as grades 1,2 , or 3 according to their degree of differentiation as assessed by morphologic criteria. ${ }^{41}$

in lichen sclerosus, were p-S6 negative (Figure 3f), indicating that this did not trigger a wound-related p-S6 response. In all three cases of basal p-S6 positive lichen sclerosus, the suprabasal cells were not stained, different from the basal-plus-parabasal or full-thickness p-S6 staining typical for differentiated VIN lesions (Figure 2). We concluded from these results that neither hyperplasia nor inflammation in the absence of cellular atypia triggers abnormal, basal p-S6. However, a minority of lichen sclerosus lesions without atypia exhibits abnormal p-S6 staining, biased in our small sample toward lesions associated with carcinoma at time of biopsy.

\section{The Relation of Laminin $\gamma^{2}$ Overexpression to Basal p-S6 Immunostaining}

Laminin $\gamma^{2}$ is not detectable immunohistochemically in the basal cells of normal epithelium but frequently is expressed abnormally by cells in the basal layer of invading fronts of many carcinomas ${ }^{21-23}$ and also by some basal cells in many oral and interfollicular epidermal dysplasias. ${ }^{24,25,29}$ Our recent study of oral dysplasias found that laminin $\gamma^{2}$ overexpression occurs solely within regions that immunostain for p-S6(ser235/236) in the basal layer. $^{29}$ We therefore asked whether laminin $\gamma^{2}$ expression is linked to basal cell S6 phosphorylation in vulvar lesions. As shown in Figures 4a and b, laminin $\gamma^{2}$ was detectable in some basal cells of two of the six differentiated VINs and five of 

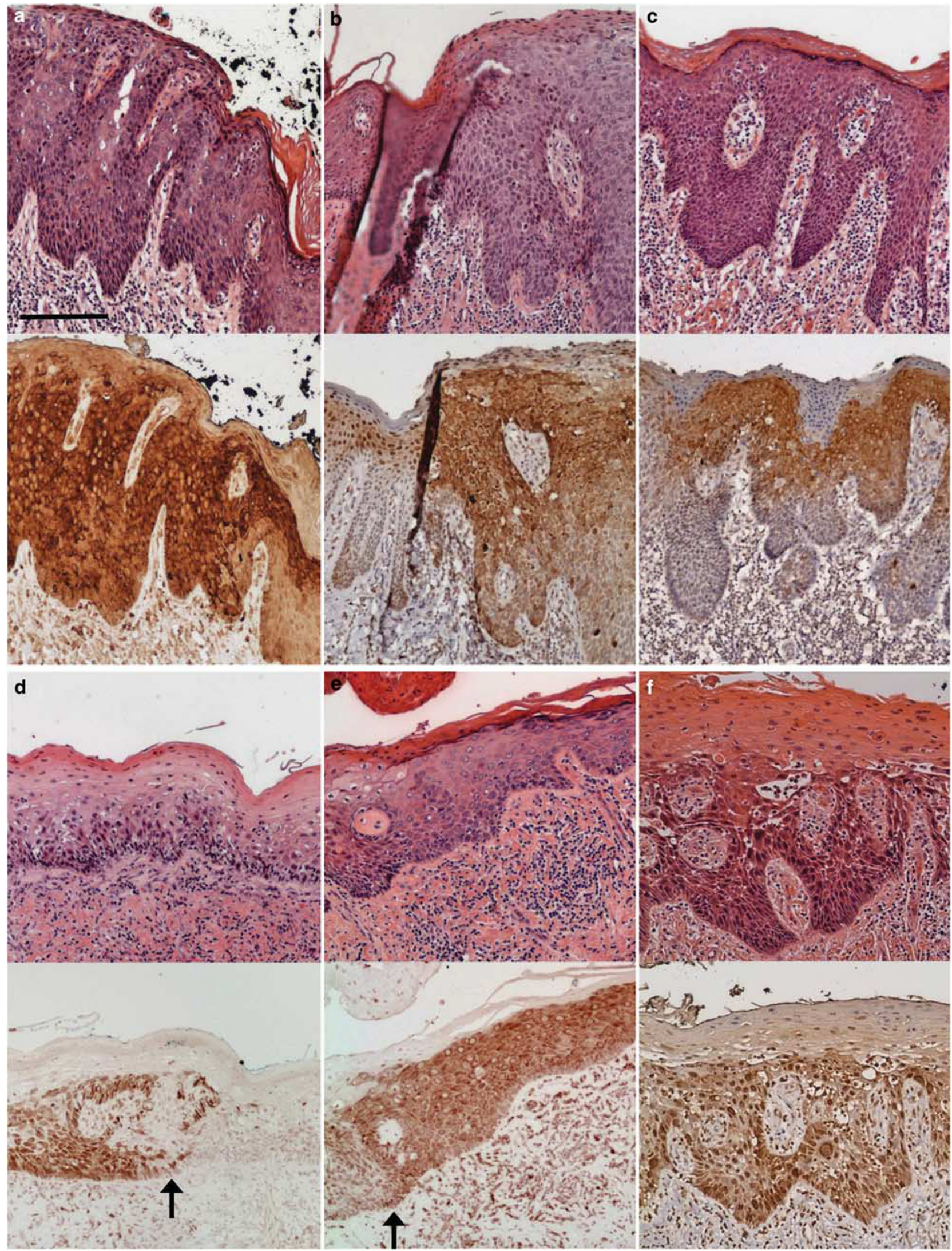

Figure 2 P-S6 immunostaining in VINs. H\&E (top) and p-S6(S235/236) (bottom) staining of classic VIN cases 8 (a), 13 (b), and 16 (c) and of differentiated VIN cases 7 (d), 2 (e), and 6 (f). Bar in a is $200 \mu \mathrm{m}$. Note full-thickness p-S6 staining in the dysplastic region at left in a, adjacent to a region with normal p-S6 staining. Note periodic short stretches of basal and parabasal p-S6 positive cells in panels b, $\mathbf{c}$ and the abrupt margin (indicated by arrows) between basal p-S6 positive and negative regions in the dysplasias shown in $\mathbf{d}$, e. 
Table 2 Summary of immunostaining results for nondysplastic vulvar lesions

\begin{tabular}{llc}
\hline Case \# & Histopathologic classification & $\begin{array}{c}\text { Basal layer } \\
p-S 6\end{array}$ \\
\hline 17 & Hyperpl, inflamm, ulcer & $\mathrm{N}$ (ulcer P) \\
18 & Hyperpl, inflamm & $\mathrm{N}$ \\
19 & Lichen sclerosus & $\mathrm{N}$ \\
20 & Lichen sclerosus & $\mathrm{N}$ \\
21 & Early lichen sclerosus & $\mathrm{N}$ \\
22 & Hyperpl & $\mathrm{N}$ \\
23 & Hyperpl & $\mathrm{N}$ (ulcer P) \\
24 & Yeast infection, severe inflamm, ulcer & $\mathrm{N}$ \\
25 & Normal & $\mathrm{N}$ \\
26 & Early lichen sclerosus/mild dysplasia* & $\mathrm{N}$ \\
27 & Lichen sclerosus & $\mathrm{P}$ \\
28 & Lichen sclerosus, hyperpl & $\mathrm{P}$ \\
29 & Lichen sclerosus (assoc. with SCC) & \\
\hline
\end{tabular}

Abbreviations: Hyperpl, hyperplasia; inflamm, inflammation; N, no p-S6-positive basal cells; P, some p-S6-positive basal cells; SCC, squamous cell carcinoma; ulcer, specimen contained an area lacking complete epithelial coverage.

Asterisk for case 26 indicates that the pathologist who originally evaluated the lesion classified it as mild dysplasia, whereas one of us (C.P.C.) detected no dysplasia in the series of sections cut from the block for H\&E and immunohistochemical staining.

the nine classic VINs tested, as well as in some areas of all invasive carcinomas (Figure 4c). As we had found for oral dysplasias, ${ }^{29}$ more cells in VINs were p-S6 positive than were laminin $\gamma^{2}$ positive. When present, laminin $\gamma^{2}$ positive cells always lay within regions of p-S6 positive basal cells (Figure 4), suggesting that events resulting in S6 phosphorylation are necessary preconditions for laminin $\gamma^{2}$ overexpression.

\section{Discussion}

The results we have described here demonstrate that abnormal phosphorylation of ribosomal protein S6 in basal epithelial cells is a readily detectable and reliable immunohistochemical biomarker of differentiated VIN lesions. Consistent with our recent findings about oral dysplasias, ${ }^{29}$ p-S6(S235/236) promises to be a very useful, single biomarker for detecting non-papillomavirus-related, potential precursors of vulvar carcinoma. Unlike classic VINs, differentiated VINs lack a consistent signature of morphologic abnormalities, so an objective biomarker would be very valuable for their diagnosis.

Squamous cell carcinomas typically have hyperactive EGFR/ERK and PI3K/AKT/mTOR signaling pathways. ${ }^{26-28,31}$ One consequence of activation of these pathways is phosphorylation of the ribosomal protein S6. S6 is a component of the $40 \mathrm{~S}$ ribosomal subunit and is thought to have a role in modulating efficiency of mRNA translation initiation. ${ }^{32}$ S6 is activated by phosphorylation at several serine residues (S235, S236, S240, and S244) near its C-terminus. ${ }^{33}$ Phosphorylation at S235 and S236 in normal keratinocytes and squamous cell carcinoma cells is accomplished by the kinase p90RSK, which is activated by the EGFR/ERK signal pathway, whereas the S240 and S244 phosphorylations appear to be accomplished exclusively by S6K1, a kinase activated by the PI3K/AKT/mTOR signaling pathway. ${ }^{29,34-36}$ An antibody specific for p-S6(S235/ 236) works well as a immunohistochemical staining reagent, whereas antibodies specific for the phosphorylated, activated states of other proteins in the EGFR/ERK and PI3K/mTOR signal pathways, which work well for western blotting, do not identify their antigens in neoplastic basal epithelial cells in formalin-fixed, paraffin-embedded tissue. ${ }^{29}$ Therefore, p-S6(S235/236) immunostaining provides the best read-out of EGFR/ERK signal pathway hyperactivity in pathology tissue specimens. ${ }^{29}$

Laminin $\gamma^{2}$ overexpression is a well-established immunohistologic marker of carcinoma ${ }^{21-23}$ and occurs also in many premalignant dysplasias of oral epithelium and epidermis. ${ }^{24,25,29}$ Recently, we reported that laminin $\gamma^{2}$ overexpression in premalignant and malignant oral and epidermal keratinocytes occurs at the level of translation and is caused by hyperactivation of the EGFR/ERK/RSK pathway, which activates both $\mathrm{S} 6$ and the translation initiation factor eIF4B. ${ }^{29}$ That study found that laminin $\gamma^{2}$ overexpression occurs solely within regions of oral dysplasias having elevated p-S6(S235/236) levels in their basal cell layer. ${ }^{29}$ The present study showed that this also is the case in VINs. P-S6 staining of VINs was more extensive than that of laminin $\gamma^{2}$ and also is a direct consequence of signal pathway hyperactivity, suggesting that $\mathrm{p}-\mathrm{S} 6$ is potentially more sensitive and valuable as an early biomarker of neoplasia than is laminin $\gamma^{2}$. An additional advantage of $\mathrm{p}-\mathrm{S} 6$ over laminin $\gamma^{2}$ is that p-S6 immunostaining does not appear to be diminished or abolished by the common histopathology service lab practice of 'baking' paraffin section slides at $50{ }^{\circ} \mathrm{C}$, unlike the great sensitivity of laminin $\gamma^{2}$ to such treatment. ${ }^{24,29}$

Biomarkers for detecting precursor lesions of vulvar squamous cell carcinoma have long been sought, especially for the often difficult to identify non-papillomavirus-related, differentiated VINs. $\mathrm{p} 16^{\mathrm{INK} 4 \mathrm{~A}}$ is a very useful marker for diagnosing high-risk papillomavirus-related epithelial neoplasias, such as virtually all cases of cervical intraepithelial neoplasia, cervical carcinoma, and classic VIN. Full or near full thickness p16 ${ }^{\text {INK4A }}$ immunostaining characterizes most classic VINs, but normal and hyperplastic vulvar epidermis, lichen sclerosus, and differentiated VIN are usually p16 negative. ${ }^{17,18}$ Presence of strongly p53-positive suprabasal cells was proposed as an aid for the diagnosis of differentiated VIN ${ }^{20}$ but this marker is not considered highly sensitive. Independent studies found a range of 54 to $83 \%$ of differentiated VINs to have abnormal p53 immunostaining 

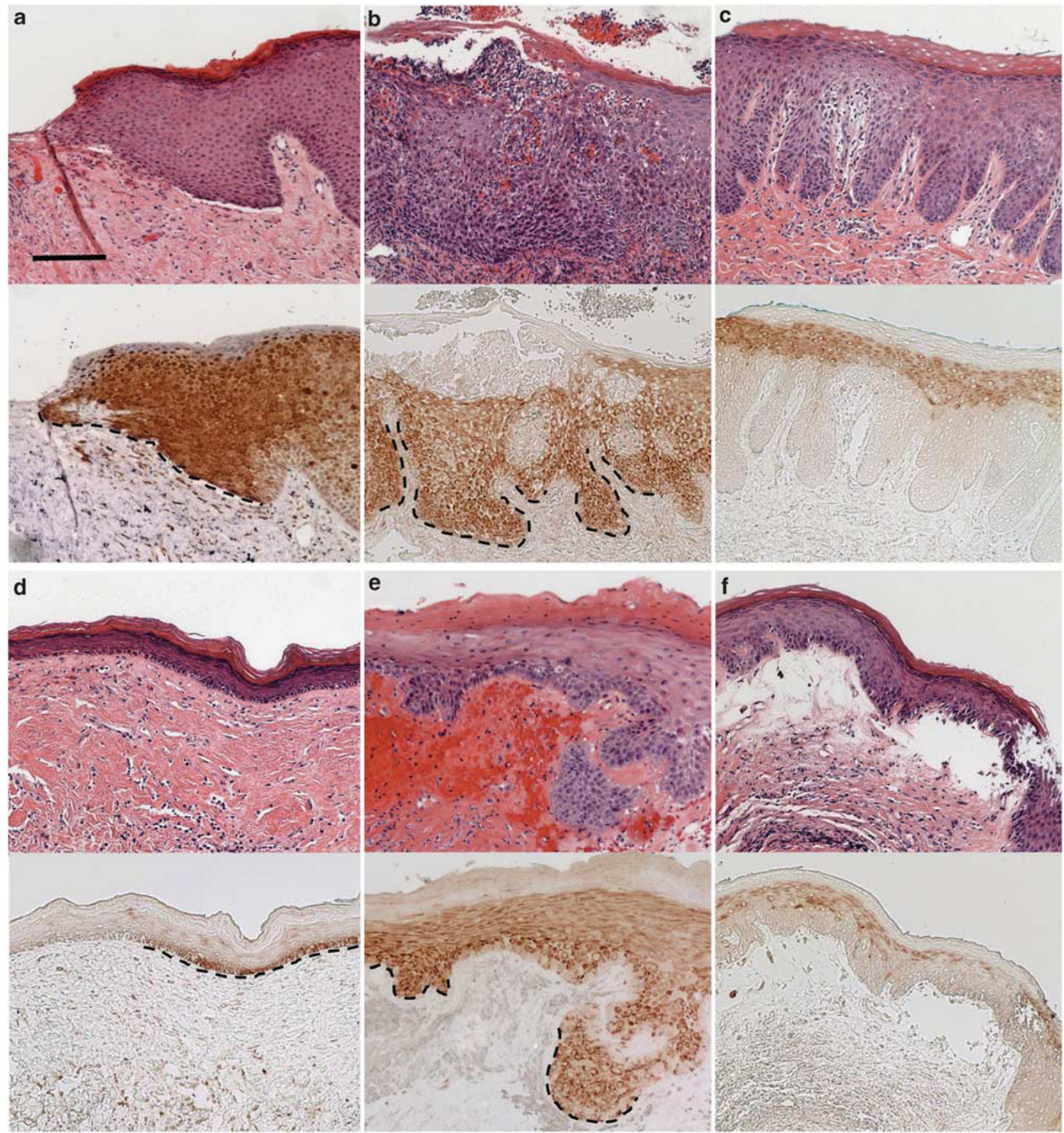

Figure 3 P-S6 positive basal cells in settings without morphologic dysplasia. H\&E staining (top) and p-S6(S235/236) immunostaining (bottom) of (a) ulcer edge in a histologically normal region of case 14, (b) severely inflamed and ulcerated region in case 24, (c) hyperplasia case 23, (d) lichen sclerosus case 29, (e) lichen sclerosus with hyperplasia case 28, (f) lichen sclerosus case 27. Bar in a is $200 \mu \mathrm{m}$. Dashed lines demarcate basal p-S6-positive regions. Note p-S6 positive basal cells at wound edge in a and in ulcerated region of c, consistent with our previous findings for wounds and ulcers in normal epidermis and oral epithelium. ${ }^{29,40}$ Note absence of apparent morphologic dysplasia or atypia in both p-S6 positive and negative regions of the lichen sclerosus lesions in $\mathbf{d}-\mathbf{f}$.

patterns..$^{9,17,20,37,38}$ The fact that p53 is detected only in some differentiated VIN lesions might be explained by differences in the type of TP53 gene mutation, with strong p53 immunostaining associated with dominant-negative missense mutations and absence of staining associated with TP53 deletions. ${ }^{9}$ Weak basal layer p53 staining has been reported in some lichen simplex chronicus and lichen sclerosus lesions in the absence of basal atypia and not associated with carcinoma. ${ }^{20}$ In these entities, any increase in p53 immunostaining intensity has been attributed to increased p53 protein levels associated with a stress response to ischemia or inflammation rather than to mutational change. ${ }^{39}$ 

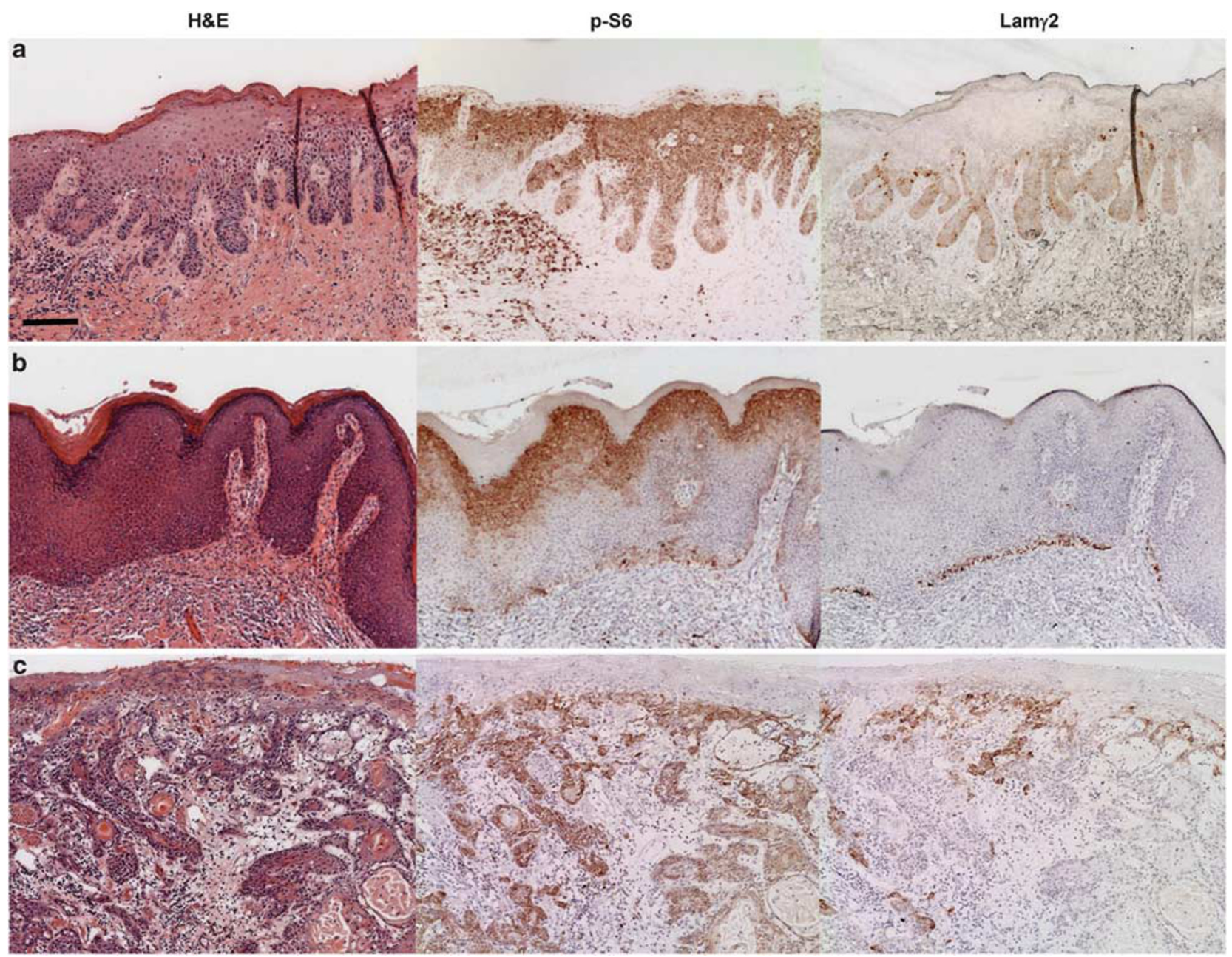

Figure 4 Relation between basal p-S6 and laminin $\gamma^{2}$ overexpression in VIN and carcinoma. H\&E staining (left), p-S6(S235/236) immunostaining (middle), and laminin $\gamma^{2}$ immunostaining (right) of (a) differentiated VIN case 1, (b) classic VIN case 9, (c) carcinoma adjacent to differentiated VIN case 4. Bar in a is $200 \mu \mathrm{m}$. Note that laminin $\gamma^{2}$ positive cells in the dysplasia and carcinoma always lay within $\mathrm{p}$-S6 positive regions, as reported previously for oral dysplasias and carcinomas. ${ }^{29}$

Two of the nine cases of classic VIN we studied did not have detectable basal p-S6, indicating that the distinctive morphologic changes including hyperplasia characteristic of classic VIN may precede activation of the EGFR/ERK signal pathway to a level sufficient to cause S6(S235/236) phosphorylation. Our study also found basal p-S6 positivity in regions of lichen sclerosus without atypia adjacent to invasive carcinoma and in one of six lichen sclerosus lesions without atypia that were not associated with known VIN or carcinoma at time of biopsy. This finding suggests the possibility that p-S6 staining detects premalignant change in lichen sclerosus lesions before the appearance of morphologic atypia. As lichen sclerosus may sometimes precede ${ }^{11,13,14}$ and be genetically linked ${ }^{8}$ to differentiated VIN, p-S6 may detect premalignant change before the appearance of morphologically detectable VIN.

In summary, our study provides evidence that p-S6(S235/236) immunostaining may be useful clinically as a biomarker of vulvar lesions at risk of progressing to invasive carcinoma. Larger retrospective studies of VIN and lichen sclerosus lesions of patients who did or did not progress to carcinoma will determine the value of $\mathrm{p}-\mathrm{S} 6$ in diagnosing vulvar premalignancy.

\section{Acknowledgements}

This research was supported by a Brigham and Women's Hospital Biomedical Research Institute grant (J.G.R.) and by postdoctoral fellowships from the Swiss National Science Foundation (Schweizerische Nationalfonds), the Novartis Foundation, and the Swiss Foundation for Grants in Biology and Medicine (Schweizerische Stiftung für BiologischMedizinische Stipendien) (M.D.).

\section{Disclosure/conflict of interest}

The authors declare no conflict of interest. 


\section{References}

1 Siegel R, Naishadham D, Jemal A. Cancer statistics, 2012. CA Cancer J Clin 2012;62:10-29.

2 Medeiros F, Nascimento AF, Crum CP. Early vulvar squamous neoplasia: advances in classification, diagnosis, and differential diagnosis. Adv Anat Pathol 2005;12:20-26.

3 Andersen WA, Franquemont DW, Williams J, et al. Vulvar squamous cell carcinoma and papillomaviruses: two separate entities? Am J Obstet Gynecol 1991;165:329-335; discussion 35-6.

4 Kurman RJ, Toki T, Schiffman MH. Basaloid and warty carcinomas of the vulva. Distinctive types of squamous cell carcinoma frequently associated with human papillomaviruses. Am J Surg Pathol 1993;17:133-145.

5 zur Hausen H. Papillomaviruses and cancer: from basic studies to clinical application. Nat Rev Cancer 2002; 2:342-350.

6 Braakhuis BJ, Tabor MP, Kummer JA, et al. A genetic explanation of Slaughter's concept of field cancerization: evidence and clinical implications. Cancer Res 2003;63:1727-1730.

7 Pinto AP, Lin MC, Mutter GL, et al. Allelic loss in human papillomavirus-positive and -negative vulvar squamous cell carcinomas. Am J Pathol 1999;154: 1009-1015.

8 Pinto AP, Lin MC, Sheets EE, et al. Allelic imbalance in lichen sclerosus, hyperplasia, and intraepithelial neoplasia of the vulva. Gynecol Oncol 2000;77: 171-176.

9 Pinto AP, Miron A, Yassin Y, et al. Differentiated vulvar intraepithelial neoplasia contains Tp53 mutations and is genetically linked to vulvar squamous cell carcinoma. Mod Pathol 2010;23:404-412.

10 Kokka F, Singh N, Faruqi A, et al. Is differentiated vulval intraepithelial neoplasia the precursor lesion of human papillomavirus-negative vulval squamous cell carcinoma? Int J Gynecol Cancer 2011;21: 1297-1305.

11 Leibowitch M, Neill S, Pelisse M, et al. The epithelial changes associated with squamous cell carcinoma of the vulva: a review of the clinical, histological and viral findings in 78 women. Br J Obstet Gynaecol 1990; 97:1135-1139.

12 Rodke G, Friedrich EG Jr., Wilkinson EJ. Malignant potential of mixed vulvar dystrophy (lichen sclerosus associated with squamous cell hyperplasia). J Reprod Med 1988;33:545-550.

13 Ueda Y, Enomoto T, Kimura T, et al. Two distinct pathways to development of squamous cell carcinoma of the vulva. J Skin Cancer 2011;2011:951250.

14 van de Nieuwenhof HP, Bulten J, Hollema $\mathrm{H}$, et al. Differentiated vulvar intraepithelial neoplasia is often found in lesions, previously diagnosed as lichen sclerosus, which have progressed to vulvar squamous cell carcinoma. Mod Pathol 2011;24:297-305.

15 Judson PL, Habermann EB, Baxter NN, et al. Trends in the incidence of invasive and in situ vulvar carcinoma. Obstet Gynecol 2006;107:1018-1022.

16 Kenter GG, Welters MJ, Valentijn AR, et al. Vaccination against HPV-16 oncoproteins for vulvar intraepithelial neoplasia. N Engl J Med 2009;361: 1838-1847.

17 Hoevenaars BM, van der Avoort IA, de Wilde PC, et al. A panel of p16 ${ }^{\text {INK4A }}$, MIB1 and p53 proteins can distinguish between the 2 pathways leading to vulvar squamous cell carcinoma. Int J Cancer 2008;123: 2767-2773.

18 Santos M, Montagut C, Mellado B, et al. Immunohistochemical staining for p16 and p53 in premalignant and malignant epithelial lesions of the vulva. Int J Gynecol Pathol 2004;23:206-214.

19 van der Avoort IA, van der Laak JA, Paffen A, et al. MIB1 expression in basal cell layer: a diagnostic tool to identify premalignancies of the vulva. Mod Pathol 2007;20:770-778.

20 Yang B, Hart WR. Vulvar intraepithelial neoplasia of the simplex (differentiated) type: a clinicopathologic study including analysis of HPV and p53 expression. Am J Surg Pathol 2000;24:429-441.

21 Koshikawa N, Moriyama K, Takamura H, et al. Overexpression of laminin $\gamma^{2}$ chain monomer in invading gastric carcinoma cells. Cancer Res 1999;59: $5596-5601$.

22 Ono Y, Nakanishi Y, Ino Y, et al. Clinocopathologic significance of laminin-5 $\gamma^{2}$ chain expression in squamous cell carcinoma of the tongue: immunohistochemical analysis of 67 lesions. Cancer 1999;85: 2315-2321.

23 Pyke C, Romer J, Kallunki P, et al. The $\gamma^{2}$ chain of kalinin/laminin 5 is preferentially expressed in invading malignant cells in human cancers. Am J Pathol 1994;145:782-791.

24 Natarajan E, Saeb M, Crum CP, et al. Co-expression of p16 $6^{\text {INK4A }}$ and laminin $5 \gamma^{2}$ by microinvasive and superficial squamous cell carcinomas in vivo and by migrating wound and senescent keratinocytes in culture. Am J Pathol 2003;163:477-491.

25 Nordemar S, Hogmo A, Lindholm J, et al. Laminin-5 $\gamma^{2}$ : a marker to identify oral mucosal lesions at risk for tumor development? Anticancer Res 2003;23: 4985-4989.

26 Dobashi Y, Suzuki S, Kimura M, et al. Paradigm of kinase-driven pathway downstream of epidermal growth factor receptor/Akt in human lung carcinomas. Hum Pathol 2010;42:214-226.

27 Einspahr JG, Calvert V, Alberts DS, et al. Functional protein pathway activation mapping of the progression of normal skin to squamous cell carcinoma. Cancer Prev Res (Phila) 2012;5:403-413.

28 Stransky N, Egloff AM, Tward AD, et al. The mutational landscape of head and neck squamous cell carcinoma. Science 2011;333:1157-1160.

29 Degen M, Natarajan E, Barron P, et al. MAPK/ERKdependent translation factor hyperactivation and dysregulated Laminin $\gamma^{2}$ expression in oral dysplasia and squamous cell carcinoma. Am J Pathol 2012;180: 2462-2478.

30 Scurry J, Wilkinson EJ. Review of terminology of precursors of vulvar squamous cell carcinoma. J Low Genit Tract Dis 2006;10:161-169.

31 Molinolo AA, Amornphimoltham P, Squarize CH, et al. Dysregulated molecular networks in head and neck carcinogenesis. Oral Oncol 2009;45:324-334.

32 Holz MK, Ballif BA, Gygi SP, et al. mTOR and S6K1 mediate assembly of the translation preinitiation complex through dynamic protein interchange and ordered phosphorylation events. Cell 2005;123: 569-580.

33 Ferrari S, Bandi HR, Hofsteenge J, et al. Mitogenactivated 70K S6 kinase. Identification of in vitro $40 \mathrm{~S}$ ribosomal S6 phosphorylation sites. J Biol Chem 1991;266:22770-22775. 
34 Anjum R, Blenis J. The RSK family of kinases: emerging roles in cellular signalling. Nat Rev Mol Cell Biol 2008;9:747-758.

35 Pende M, Um SH, Mieulet V, et al. S6K1(-/-)/ S6K2 $(-/-)$ mice exhibit perinatal lethality and rapamycin-sensitive 5 -terminal oligopyrimidine mRNA translation and reveal a mitogen-activated protein kinase-dependent S6 kinase pathway. Mol Cell Biol 2004;24:3112-3124.

36 Roux PP, Shahbazian D, Vu H, et al. RAS/ERK signaling promotes site-specific ribosomal protein S6 phosphorylation via RSK and stimulates cap-dependent translation. J Biol Chem 2007;282:14056-14064.

37 Mulvany NJ, Allen DG. Differentiated intraepithelial neoplasia of the vulva. Int J Gynecol Pathol 2008; 27:125-135.
38 Aulmann S, Schleibaum J, Penzel R, et al. Gains of chromosome region 3q26 in intraepithelial neoplasia and invasive squamous cell carcinoma of the vulva are frequent and independent of HPV status. J Clin Pathol 2008;61:1034-1037.

39 Liegl B, Regauer S. p53 immunostaining in lichen sclerosus is related to ischaemic stress and is not a marker of differentiated vulvar intraepithelial neoplasia (d-VIN). Histopathology 2006;48:268-274.

40 Natarajan E, Omobono JD 2nd, Guo Z, et al. A keratinocyte hypermotility/growth-arrest response involving Laminin 5 and $\mathrm{p} 16^{\mathrm{INK} 4 \mathrm{~A}}$ activated in wound healing and senescence. Am J Pathol 2006;168:1821-1837.

41 Wilkinson EJ. Normal histology and nomenclature of the vulva, and malignant neoplasms, including VIN. Dermatol Clin 1992;10:283-296. 